BULLETIN OF THE

AMERICAN MATHEMATICAL SOCIETY

Volume 78, Number 5, September 1972

\title{
FOLIATIONS AND THE PROPAGATION OF ZEROES OF SOLUTIONS OF PARTIAL DIFFERENTIAL EQUATIONS
}

\author{
BY E. C. ZACHMANOGLOU ${ }^{1}$
}

Communicated by François Treves, March 20, 1972

1. Introduction. Let $P(x, D)$ be a linear partial differential operator of order $m$ with complex-valued coefficients defined and analytic in an open connected set $\Omega$ in $R^{n}$,

$$
P(x, D)=\sum_{|\alpha| \leqq m} a^{\alpha}(x) D^{\alpha},
$$

with the usual notation. The principal part $P_{m}(x, D)$ is the homogeneous part of $P(x, D)$ of order $m$. At a fixed point $x \in \Omega$, the (real) zeroes of $P_{m}(x, \xi)$ form a cone in $R^{n}$ which is called the (real) characteristic cone of $P(x, D)$ at $x$. We will denote by $\mathscr{A}$ the ring of real-valued analytic functions in $\Omega$.

In this paper we consider partial differential operators having the following property: There exist $r$ analytic vector fields in $\Omega$,

$$
A_{j}=\sum_{i=1}^{n} a_{j}^{i} D_{i}, \quad j=1, \ldots, r,
$$

with $a_{j}^{i} \in \mathscr{A}, i=1, \ldots, n, j=1, \ldots, r$, such that at each point of $\Omega$ the characteristic cone of $P(x, D)$ is orthogonal to every $A_{j}$. More precisely, we assume that, for every $x \in \Omega$,

$$
P_{m}(x, \xi)=0, \quad \xi \in R^{n} \Rightarrow \sum_{i=1}^{n} a_{j}^{i}(x) \xi_{i}=0, \quad j=1, \ldots, r .
$$

We will denote by $\mathscr{L}\left(A_{1}, \ldots, A_{r}\right)$ the Lie algebra generated by $A_{1}, \ldots, A_{r}$, i.e. the smallest set of analytic vector fields in $\Omega$ which is closed under the operations of taking brackets and linear combinations with coefficients in $\mathscr{A}$.

According to a theorem of Nagano [1], the Lie algebra $\mathscr{L}\left(A_{1}, \ldots, A_{r}\right)$ defines a unique partition of $\Omega$ into maximal integral manifolds of $\mathscr{L}\left(A_{1}, \ldots, A_{r}\right)$, that is, $\Omega$ is the disjoint union of maximal integral manifolds of $\mathscr{L}\left(A_{1}, \ldots, A_{r}\right)$. This partition is called a foliation and each maximal integral manifold is called a leaf of the foliation.

\footnotetext{
AMS 1970 subject classifications. Primary 35A05; Secondary 58A30.

Key words and phrases. Leaves of foliations, integral manifolds, propagation of zeroes of solutions, linear homogeneous partial differential equations.

1 Research supported by NSF Grant GP-20547.
} 
In this paper we prove that the zeroes of solutions of the equation $P(x, D) u=0$ propagate along the leaves of the foliation defined by $\mathscr{L}\left(A_{1}, \ldots, A_{r}\right)$. More precisely let $u$ be a distribution solution of $P(x, D) u$ $=0$ in $\Omega$ and suppose that $u$ vanishes in an open neighborhood of a point $x \in \Omega$. Then $u$ also vanishes in an open neighborhood of every point of the leaf through $x$ of the foliation defined by $\mathscr{L}\left(A_{1}, \ldots, A_{r}\right)$.

This result includes the well-known result on the propagation of zeroes of solutions of elliptic equations. It includes also the result of Bony [2] concerning degenerate elliptic second order equations and the result of Zachmanoglou [3] concerning first order equations with complex-valued coefficients.

2. Some results on foliations. The bracket of two vector fields $A$ and $B$ is the commutator $[A, B]=A B-B A$. If $A$ and $B$ have analytic coefficients in $\Omega$, then $[A, B]$ is also a vector field with analytic coefficients in $\Omega$. The (real) vector space of all real analytic vector fields in $\Omega$, equipped with the bracket operation, is a Lie algebra denoted by $\mathscr{L}(\Omega)$. It is also a module over the ring $\mathscr{A} . \mathscr{A}\left(A_{1}, \ldots, A_{r}\right)$ will denote the smallest $\mathscr{A}$-submodule of $\mathscr{L}(\Omega)$ containing the vector fields $A_{1}, \ldots, A_{r}$. A vector subspace of $\mathscr{L}(\Omega)$ which is closed under the bracket operation is a Lie subalgebra of $\mathscr{L}(\Omega) . \mathscr{L}\left(A_{1}, \ldots, A_{r}\right)$ is the smallest $\mathscr{A}$-submodule and Lie subalgebra of $\mathscr{L}(\Omega)$ containing $A_{1}, \ldots, A_{r}$.

Let $\mathscr{L}$ be a vector subspace of $\mathscr{L}(\Omega)$. For any $x \in \Omega$ we set $\mathscr{L}(x)$ $=\{A(x): A \in \mathscr{L}\} . \mathscr{L}(x)$ is a subspace of $R^{n}$ and is called the integral element of $\mathscr{L}$ at $x$. An integral manifold $N$ of $\mathscr{L}$ is a connected submanifold of $\Omega$ such that, for every $x \in N$, the tangent space to $N$ at $x$ is equal to $\mathscr{L}(x)$.

TheOREM 1 (NAgANo). If $\mathscr{L}$ is a Lie subalgebra of $\mathscr{L}(\Omega)$, then through every point $x \in \Omega$ passes a maximal integral manifold $L^{x}$ of $\mathscr{L}$. Any integral manifold of $\mathscr{L}$ containing $x$ is an open submanifold of $L^{x}$.

According to Theorem $1, \mathscr{L}$ defines a unique partition of $\Omega$ by maximal integral manifolds of $\mathscr{L}$ (that is, $\Omega$ is the disjoint union of maximal integral manifolds of $\mathscr{L}$ ). This partition of $\Omega$ will be called the foliation defined by $\mathscr{L}$ and each maximal integral manifold will be called a leaf of the foliation. Note that, for every $x \in \Omega$, the dimension of the leaf $L^{x}$ containing $x$ is equal to the dimension of the integral element $\mathscr{L}(x)$.

With the additional assumption that $\operatorname{dim} \mathscr{L}(x)$ is constant in $\Omega$, Theorem 1 is the classical theorem of Frobenius (see Chevalley [4]). However, the Frobenius theorem is also valid in the $C^{\infty}$ case. Theorem 1 was proved by Nagano [1] and it is not generally valid in the $C^{\infty}$ case.

An integral curve of an analytic vector field, say $A=\sum a^{i} D_{i}$, is a solution $x=x(t)$ of the system 


$$
d x_{i} / d t=a^{i}(x), \quad i=1, \ldots, n .
$$

A trajectory of a collection $\mathscr{C}$ of analytic vector fields in $\Omega$ is a continuous, piecewise analytic curve in $\Omega$, each analytic piece of which is an integral curve of a member of $\mathscr{C}$.

TheOREm 2 (Chow-HermanN). Let $\mathscr{L}$ be a (real) vector subspace of $\mathscr{L}(\Omega)$ and suppose that the smallest $\mathscr{A}$-submodule and Lie subalgebra of $\mathscr{L}(\Omega)$ containing $\mathscr{L}$ is equal to $\mathscr{L}(\Omega)$. Then, for every point $x \in \Omega$, the set of points of $\Omega$ that can be connected to $x$ by trajectories of $\mathscr{L}$ is equal to $\Omega$.

A weaker form of this theorem was first proved by Chow [5]. A proof of Theorem 2 (with $\Omega$ being any differentiable manifold) is given in the book of Hermann [6, Chapter 18]. Incidentally Theorem 2 is also valid in the $C^{\infty}$ case.

We apply now Theorem 1 to the Lie subalgebra $\mathscr{L}\left(A_{1}, \ldots, A_{r}\right)$. The leaf of its foliation passing through the point $x$ will be denoted by $L^{x}\left(A_{1}, \ldots, A_{r}\right)$. We also apply Theorem 2 to the vector subspace $\mathscr{A}\left(A_{1}, \ldots, A_{r}\right)$ of $\mathscr{L}(\Omega)$. By restricting to the leaf $L^{x}\left(A_{1}, \ldots, A_{r}\right)$ Theorem 2 yields

THEOREM 3. Let $x$ be any point of $\Omega$. The set of points of $\Omega$ that can be connected to $x$ by trajectories of $\mathscr{A}\left(A_{1}, \ldots, A_{r}\right)$, is equal to the leaf $L^{x}\left(A_{1}, \ldots, A_{r}\right)$.

\section{The propagation of zeroes.}

LeMma 1. Let $A=\sum_{i=1}^{n} a^{i} D_{i}$ be an analytic vector field in $\Omega$ such that at each point of $\Omega$ the characteristic cone of $P(x, D)$ is orthogonal to $A$, that is, for every $x \in \Omega$,

$$
P_{m}(x, \xi)=0, \quad \xi \in R^{n} \Rightarrow \sum_{i=1}^{n} a^{i}(x) \xi_{i}=0 .
$$

Let $u$ be a distribution solution of $P(x, D) u=0$ in $\Omega$ and suppose that $u$ vanishes in an open neighborhood of some point $x$ of $\Omega$. Then $u$ vanishes in an open neighborhood of every point of the integral curve of $A$ passing through $x$.

The proof of this lemma consists of locally straightening out the vector field $A$ and applying Theorem 1 of [7] concerning the propagation of zeroes of solutions of partial differential equations with flat characteristic cones. Theorem 1 of [7] was proved using Holmgren's uniqueness theorem as extended to distribution solutions by Hörmander [8] and a method first used by John [9]. 
It follows immediately from Lemma 1 that a solution of $P(x, D) u=0$ which vanishes in a neighborhood of any point $x \in \Omega$ also vanishes in a neighborhood of every point of a trajectory of $\mathscr{A}\left(A_{1}, \ldots, A_{r}\right)$ containing $x$. Combining this with Theorem 3 we obtain the main result of this paper.

THEOREM 4. Let $P(x, D)$ be a partial differential operator with analytic coefficients in an open connected set $\Omega$ of $R^{n}$. Suppose that there are r analytic vector fields $A_{1}, \ldots, A_{r}$ in $\Omega$ such that at each point of $\Omega$ the characteristic cone of $P(x, D)$ is orthogonal to every $A_{j}$, i.e. for every $x \in \Omega$ condition (3) is satisfied. Let $u$ be a distribution solution of $P(x, D) u=0$ in $\Omega$, and suppose that $u$ vanishes in an open neighborhood of some point $x \in \Omega$. Then $u$ vanishes in a neighborhood of every point of the leaf $L^{x}\left(A_{1}, \ldots, A_{r}\right)$ of the foliation defined by $\mathscr{L}\left(A_{1}, \ldots, A_{r}\right)$.

COROLLARY 1. Under the assumption of Theorem 4 and if, in addition, at every point of $\Omega$ the dimension of the integral element of $\mathscr{L}\left(A_{1}, \ldots, A_{r}\right)$ is equal to $n$, then every solution of $P(x, D) u=0$ vanishing in a neighborhood of a point of $\Omega$ must vanish in the whole of $\Omega$.

4. Some examples. If $P(x, D)$ is an elliptic operator in $\Omega$, then, by definition, for every $x \in \Omega$,

$$
P_{m}(x, \xi)=0, \quad \xi \in R^{n} \Leftrightarrow \xi=0 .
$$

In this case we can take $A_{j}=D_{j}, j=1, \ldots, n$. We have $\mathscr{L}\left(D_{1}, \ldots, D_{n}\right)$ $=\mathscr{L}(\Omega)$ and its foliation consists of a single leaf, the whole of $\Omega$. Hence any solution of $P(x, D) u=0$ vanishing in a neighborhood of a point of $\Omega$ must vanish in the whole of $\Omega$.

The operators studied by Bony [2] are of the form

$$
P(x, D)=\sum_{j=1}^{r} A_{j}^{2}+B+c
$$

where $A_{1}, \ldots, A_{r}$ and $B$ are real first order operators with analytic coefficients in $\Omega$. At every point of $\Omega$ the characteristic cone is orthogonal to the vector fields $A_{1}, \ldots, A_{r}$. Under the assumption that the dimension of the integral element of $\mathscr{L}\left(A_{1}, \ldots, A_{r}\right)$ is equal to $n$ at every point of $\Omega$, Bony showed that any solution of $P(x, D) u=0$ vanishing in a neighborhood of a point of $\Omega$ must vanish in the whole of $\Omega$. This of course is precisely the assertion of Corollary 1.

A first order operator with complex-valued coefficients is of the form

$$
P(x, D)=A+i B+c
$$

where $A$ and $B$ are real first order operators. The characteristic cone is 
orthogonal to the vector fields $A$ and $B$. The propagation of zeroes and uniqueness in the Cauchy problem for this operator were studied in [3].

\section{BIBLIOGRAPHY}

1. T. Nagano, Linear differential systems with singularities and an application to transitive Lie algebras, J. Math. Soc. Japan 18 (1966), 398-404. MR 33 \# 8005.

2. Jean-Michel Bony, Principe du maximum et inégalité de Harnack pour les opérateurs elliptiques dégénérés, Séminaire de Théorie du Potentiel, dirigé par M. Brelot, G. Choquet et J. Deny: 1967/68, Exposé 10, Secrétariat mathématique, Paris, 1969. MR 42 \# 3412.

3. E. C. Zachmanoglou, Propagation of zeroes and uniqueness in the Cauchy problem for first order partial differential equations, Arch. Rational Mech. Anal. 38 (1970), 178-188. MR 41 \# 5769.

4. C. Chevalley, Theory of Lie groups. Vol. 1, Princeton Math. Series, vol. 8, Princeton Univ. Press, Princeton, N.J., 1946. MR 7, 412.

5. W. L. Chow, Uber Systeme von linearen partiellen Differentialgleichungen erster Ordnung, Math. Ann 117 (1939), 98-105. MR 1, 313.

6. R. Hermann, Differential geometry and the calculus of variations, Math. in Sci. and Engineering, vol. 49, Academic Press, New York, 1968. MR 38 \#1635.

7. E. C. Zachmanoglou, An application of Holmgren's theorem and convexity with respect to differential operators with flat characteristic cones, Trans. Amer. Math. Soc. 140 (1969), 109-115. MR 39 \# 1790.

8. L. Hörmander, Linear partial differential operators, Die Grundlehren der math. Wissenschaften, Band 116, Academic Press, New York; Springer-Verlag, Berlin, 1963. MR 28 \# 4221.

9. F. John, On linear partial differential equations with analytic coefficients. Unique continuation of data, Comm. Pure Appl. Math. 2 (1949), 209-253. MR 12, 185.

Department of Mathematics, Purdue University, Lafayette, Indiana 47907 